\title{
CORRELAÇÃO DA COMPOSIÇÃO CORPORAL COM A INCIDÊNCIA DE DOENÇAS CARDIOVASCULARES EM UM HOSPITAL DO OESTE DO PARANÁ
}

\section{CORRELATION OF BODY COMPOSITION WITH THE INCIDENCE OF CARDIOVASCULAR DISEASES IN A HOSPITAL IN THE WEST OF PARANÁ}

\author{
Bruno Eduardo Malanchen ${ }^{1}$, Flavio Aparecido da Silva², Daniela Miotto Bernardi³ \\ Debora Regina Rendes Poletto Pappen ${ }^{*}$, Dinei Prado Filho ${ }^{5}$
}

\begin{abstract}
${ }^{1}$ Profissional de Educação Física, Nutricionista e especialista em Medicina Esportiva. ${ }^{2}$ Profissional de Educação Física e acadêmico de Nutrição do Centro Universitário FAG. ${ }^{3}$ Nutricionista, Doutora em Alimentos e Nutrição e Professora do Centro Universitário FAG. ${ }^{4}$ Nutricionista, Mestre em Engenharia de Alimentos e Professora do Centro Universitário FAG. ${ }^{5}$ Profissional de Educação Física e Mestre em Biociências e Saúde.

*Autor correspondente: de poletto@hotmail.com, https://orcid.org/0000-0003-0643-0346
\end{abstract}

DOI: 10.35984/fjh.v2i2.199

\begin{abstract}
RESUMO
Vários são os fatores de risco para doenças cardiovasculares, como por exemplo: sobrepeso/obesidade, sedentarismo, estresse, fatores genéticos, hipertensão arterial, tabagismo, idade, diabetes mellitus, dislipidemias, dentre outros. A partir dessa prerrogativa, o objetivo do trabalho foi analisar a composição corporal e a incidência de doenças cardiovasculares ou doenças crônicas não transmissíveis associadas às mesmas, de indivíduos adultos internados em um hospital no oeste do Paraná. A pesquisa se utilizou de dados coletados de prontuários de pacientes de ambos os sexos, com idade entre 40 e 90 anos, que apresentavam doenças cardiovasculares ou doenças crônicas não transmissíveis. Além da incidência de doenças, os dados de composição corporal como peso, altura e dobras cutâneas, também foram coletados. Os resultados foram expressos por meio de estatística descritiva e quantitativa e analisados pelos testes de Shapiro-Wilk., Mann-Whitney-U, teste T, Qui-quadrado, ANOVA e pelo teste de Correlação de Spearman com o grau de significância de 5\%. A maior incidência patológica nos indivíduos era de hipertensão arterial. Os resultados mostraram que quanto mais a idade avança, menor é o peso do indivíduo e também a circunferência do braço. Ainda, indivíduos com menor Estatura demonstraram possuir maiores valores de Prega Cutânea Tricipital. O índice de massa corporal e o peso apresentaram maiores médias para quem possuía até duas patologias associadas. No entanto, possuir uma ou mais patologias não diferiu entre indivíduos avaliados para estas variáveis. Essas doenças fazem parte das principais causas de morbidade e mortalidade no mundo e mudanças de peso e maior acúmulo de gordura corporal aumentam o risco para o seu acontecimento.
\end{abstract}

Palavras-chave: Antropometria, Doenças Cardiovasculares, Composição Corporal, Obesidade, Doenças Crônicas.

\begin{abstract}
There are several risk factors for cardiovascular diseases, such as: overweight / obesity, physical inactivity, stress, genetic factors, arterial hypertension, smoking, age, diabetes mellitus, dyslipidemia, among others. Based on this prerogative, the objective of the study was to analyze body composition and the incidence of cardiovascular diseases or chronic noncommunicable diseases associated with them, of adult individuals admitted to a hospital in western Paraná. The research used data collected from medical records of patients of both sexes, aged between 40 and 90 years, who had cardiovascular diseases or chronic non-
\end{abstract}


communicable diseases. In addition to the incidence of disease, data on body composition such as weight, height and skin folds were also collected. The results were expressed using descriptive and quantitative statistics and analyzed using the Shapiro-Wilk., Mann-Whitney-U tests, T test, Chi-square test, ANOVA and the Spearman correlation test with a 5\% significance level. The highest pathological incidence in individuals was arterial hypertension. The results showed that the more the age advances, the smaller the individual's weight and also the arm circumference. Still, individuals with shorter stature showed higher values of tricipital skinfold. The body mass index and weight showed higher averages for those who had up to two associated pathologies. However, having one or more pathologies did not differ between individuals assessed for these variables. These diseases are part of the main causes of morbidity and mortality in the world and changes in weight and greater accumulation of body fat increase the risk for their occurrence.

Keywords: Anthropometry, Cardiovascular Disease, Body Composition, Obesity, Cronic Disease.

\section{INTRODUÇÃO}

A obesidade vem se tornando um dos mais sérios problemas de saúde pública no Brasil e no Mundo, pois a sua prevalência vem aumentando significativamente nas últimas décadas (OGDEN et al., 2012; IBGE, 2010; WANG, MONTEIRO E POPKIN, 2002). De acordo com a Organização Mundial de Saúde, a obesidade é caracterizada como sendo um acúmulo anormal ou excessivo de gordura, o que pode prejudicar a saúde e aumentar o risco para o desenvolvimento de inúmeras doenças (World Health Organization, 2015).

Evidências indicam que a distribuição de gordura é um dos principais determinantes do risco cardiovascular em indivíduos com peso normal, com sobrepeso e moderadamente obesos, porém, considera-se que medidas de obesidade e diferenças fisiológicas entre os sexos também devam ser consideradas como fatores que possivelmente podem colaborar com a incidência desse tipo de doença, as vezes independentemente da quantidade de gordura corporal dos indivíduos (LARSSON et al., 1984; SCHNEIDER et al., 2010; YUSUF et al., 2005; VAN et al., 2009; WANG, 2008; GEER \& SHEN, 2009).

Além disso, a obesidade também é um importante fator de risco para o desenvolvimento de doença arterial coronariana (DAC), que confere um grau de risco cardiovascular semelhante ao associado à hipertensão, hiperlipidemia, tabagismo e sedentarismo (MELANSON et al., 2001). Existem evidências de que as medidas de classificação de obesidade geral e abdominal também são preditores de rigidez arterial em pacientes com obesidade mórbida, aumentando então o risco para que se desenvolva doença arterial coronária (NORDSTRAND et al., 2011).

Pessoas obesas tendem a ter um acúmulo de gordura nas vísceras corporais, aumentando ainda mais o risco para desenvolver problemas secundários. Como o tecido adiposo também se acumula nas vísceras, o mesmo também tem uma associação conhecida com complicações metabólicas e com anormalidades de fatores que regulam as quantidades circulantes de glicose, insulina e lipídios. Consequentemente, quanto maiores os estoques de gordura visceral, maiores os riscos de possíveis doenças cardíacas, coronarianas e doenças crônicas não transmissíveis, em geral (MAURIĖGE et al., 2000; TATSUKAWA et al., 2000).

As DCNTs também podem ser consideradas um problema de saúde pública no Brasil e no mundo, sendo responsáveis diretamente ou de maneira associada, por um grande número de óbitos (MINISTÉRIO DA SAÚDE, 2013; WHO, 2013; MENDIS et al., 2011). 
As medidas corporais antropométricas têm sido utilizadas para avaliar a distribuição e o acúmulo de gordura corporal. Dentre essas medidas estão o índice de massa corporal (IMC), a circunferência da cintura (CC) e relação cintura-quadril (RCQ), além da aferição de pregas cutâneas (prega cutânea bicipital, prega cutânea abdominal, prega cutânea tricipital, dentre outras). No entanto, essas medidas mostraram correlacionar-se diferentemente com 0 risco de Doenças Cardiovasculares. Embora o IMC seja usado frequentemente como uma medida de adiposidade em estudos epidemiológicos, entende-se que o uso do IMC sozinho não é a medida mais precisa do aumento do risco de DCV, portanto, correlacionar diversos marcadores de composição corporal seria fundamental para correlacionar a mesma com a incidência desse tipo de patologia, evidenciando seus demais fatores de risco (MARGARET \& SHIUN, 2005; ARAUJO, 2017; SANTANA et al., 2016; LEE, 2008).

Nesse sentido, o objetivo deste estudo será verificar a prevalência de doenças cardiovasculares e seus preditores de risco, correlacionando-os com as medidas de composição corporal.

\section{METODOLOGIA}

A pesquisa cumpriu com os procedimentos éticos de acordo com as "Diretrizes e Normas Regulamentadoras de Pesquisa Envolvendo Seres Humanos" nas resoluções 466/12 e no 510/16, bem como da Norma Operacional CNS no 001/2013, editada pela Comissão Nacional de Saúde e aprovada pelo Comitê de Ética com Seres Humanos, do Centro Universitário Assis Gurgacz, sob o parecer de número 3.391.436 e registro do CAAE: 13564219.8 .0000 .5219$.

O estudo foi de característica descritiva, sendo realizado de maneira transversal, com dados coletados de prontuários já existentes, de 86 pacientes de ambos os sexos, com idade entre 40 e 90 anos, já internados, no ano de 2019, em um hospital da cidade de Cascavel, Paraná. Foram incluídos no projeto os dados de pacientes que tinham doenças cardiovasculares ou preditores de risco, respectivamente, desse tipo de patologia e foram excluídos da pesquisa os dados de pacientes que não se encaixaram nessas características. As doenças avaliadas foram: Doença arterial coronariana (DAC) (analisada juntamente com pacientes que tinham a intervenção de angioplastia), hipertensão arterial sistêmica (HAS), infarto agudo do miocárdio (IAM), insuficiência cardíaca (IC) e dislipidemias. As variáveis antropométricas analisadas foram: Peso, estatura, índice de massa corporal (IMC), prega cutânea biciptal (PCB), prega cutânea tricipital (PCT), circunferência muscular do braço $(\mathrm{CMB})$, circunferência do braço $(\mathrm{CB})$. Propôs-se ao Comitê de Ética e Pesquisa com Seres Humanos que os termos de Consentimento Livre e Esclarecido e Assentimento fossem dispensados, pois foi realizada a coleta de dados de prontuários já existentes no hospital, não havendo contato com os pacientes nem a coleta dos dados pessoais dos mesmos, conforme Termo de Uso de Dados.

A coleta de dados para a pesquisa foi realizada em um Hospital da Cidade de Cascavel, Paraná, por meio de um sistema interno de armazenamento de prontuários, contidos no sistema de Prontuário digital do hospital, como citado anteriormente, conforme autorização da instituição. Após a coleta, os dados obtidos foram tabulados em planilhas de Excel, em uma Instituição de Ensino privada da mesma cidade. Os resultados foram expressos por meio da estatística descritiva e analisados quanto à normalidade dos resíduos, através do teste de Shapiro-Wilk. Para comparar as variáveis quantitativas entre os sexos, foi empregado o teste de Mann-Whitney-U e o teste $\mathrm{T}$, conforme a distribuição de curva. O teste de Qui-quadrado para 
independência foi utilizado para verificar a distribuição de frequências entre os sexos e o número de patologias observados. A ANOVA de uma via foi adotada para comparar as variáveis quantitativas em relação ao número de patologias observadas. A correlação de Spearman foi aplicada para associar as variáveis quantitativas. $O$ programa R Version 1.1.453 - (c) 2009-2018, Inc. foi utilizado para realizar os testes. Valores considerados significativos foram de $5 \%$.

\section{RESULTADOS E DISCUSSÃO}

O presente estudo contou com a participação de 86 pacientes, com idade entre 40 e 90 anos. A Tabela 1 apresenta, de acordo com a sua frequência relativa e absoluta, a amostra que era composta de pacientes portadores de patologias associadas a doenças cardiovasculares, ou ainda, pacientes que passaram por intervenção clínica de angioplastia.

Tabela 1. Valores de frequência relativa e absoluta para os casos de doenças e intervenções encontradas na amostra.

\begin{tabular}{lcc}
\hline Patologias/intervenções & N & $\%$ \\
\hline HAS & 69 & 80,2 \\
Dislipidemias & 21 & 24,4 \\
Angioplastia & 13 & 15,1 \\
IC & 12 & 13,9 \\
IAM & 7 & 8,1 \\
DAC & 6 & 7,0 \\
\hline
\end{tabular}

HAS: Hipertensão Arterial Sistêmica; IC: Insuficiência Cardíaca; IAM: Infarto Agudo do Miocárdio; DAC: Doença Arterial Coronariana.

Coelho el al. (2018) avaliaram as causas de morbidade em pessoas com mais de 50 anos de idade. Esse estudo avaliou 356 pacientes e suas causas subjacentes de morte, com média de idade em torno de 70 anos, observando que em $62,9 \%$ dos casos os pacientes possuíam hipertensão antes de falecer. O estudo também avaliou outras comorbidades relatadas, sendo que dentre elas apresentaram-se a doença arterial coronariana (DAC) (18,8\%), insuficiência cardíaca (IC) $(14,0 \%)$ e acidente vascular cerebral (12,9\%) (também correlacionada às patologias cardiovasculares). $\mathrm{Na}$ amostra do presente estudo, observou-se um número relativamente maior para a ocorrência de Hipertensão Arterial Sistêmica em relação às demais patologias, sendo que $80,2 \%$ dos pacientes eram diagnosticados com essa patologia, bem como DAC se mostrou presente em $7 \%$ e IC em $13,9 \%$ dos casos.

O teste de Qui-Quadrado foi utilizado para demonstrar a frequência absoluta entre os sexos em relação ao número de patologias e/ou intervenções. Os resultados obtidos estão na tabela 2.

Tabela 2. Valores de frequência absoluta entre os sexos em relação ao número de patologias e intervenções observadas nos pacientes

\begin{tabular}{lcccc} 
& \multicolumn{4}{c}{ Número de casos patológicos/intervenções } \\
Sexo & Uma & Duas & Três & p-valor \\
\hline Masculino $(\mathbf{n}=\mathbf{5 7})$ & 21 & 25 & 11 & \multirow{2}{*}{0,165} \\
Feminino $(\mathbf{n = 2 9})$ & 9 & 9 & 11 & \\
\cline { 1 - 4 } TOTAL & 30 & 34 & 22 & \\
\hline
\end{tabular}

De acordo com a tabela 2, verificamos que não houve desajuste de frequências para os sexos em relação ao número de patologias e intervenções verificadas nos pacientes, ou seja, tanto o sexo masculino quanto o feminino 
apresentam frequências iguais para apresentar uma, duas ou três doenças e/ou intervenções (X-squared $=3,594 ; \quad \mathrm{p}=0,165)$. Portanto, 0 número de patologias/intervenções entre os sexos foi igual.

Coelho et al. (2018), já citados anteriormente, demostraram, em relação aos sexos, que as mulheres tiveram probabilidade de hipertensão quase duas vezes mais altas do que os homens. Outro estudo realizado no Brasil encontrou uma diferença entre os sexos apenas na faixa etária acima de 70 anos $(82,4 \%$ em mulheres vs. $57,1 \%$ em homens, $p<0,05$ ) (CESARINO et al., 2008). Da mesma forma, a American Heart Association (AHA) identificou maior prevalência de hipertensão entre as mulheres com idade igual ou superior a 65 anos, em comparação com homens da mesma idade (MOZAFFARIAN et al., 2015).

$\mathrm{Na}$ tabela 3, são expressos os valores de média e desvio padrão para as variáveis quantitativas Idade, Peso, Estatura, IMC, PCB, CB, PCT e CMB, relacionadas à composição corporal, separando os pacientes pelo sexo, por meio dos testes T e teste de Mann-Whitney-u.

Tabela 3. Valores expressos em média e desvio padrão para as variáveis quantitativas separadas por sexo.

\begin{tabular}{|c|c|c|c|c|}
\hline Variáveis & $\begin{array}{l}\text { Masculino } \\
(n=57)\end{array}$ & $\begin{array}{c}\text { Feminino } \\
(n=29)\end{array}$ & p-valor & $\begin{array}{c}\text { Ambos os } \\
\text { Sexos }(n=86)\end{array}$ \\
\hline Idade & $67,84 \pm 11,78$ & $73,21 \pm 10,45$ & $0,035^{\star}$ & $69,65 \pm 11,57$ \\
\hline Peso (Kg) & $82,13 \pm 16,35$ & $70,25 \pm 11,81$ & $<0,001^{\star \star \star}$ & $78,13 \pm 15,94$ \\
\hline Estatura $(\mathrm{Cm})$ & $172,20 \pm 7,51$ & $159,5 \pm 5,85$ & $<0,001^{\star \star \star *}$ & $167,90 \pm 9,21$ \\
\hline IMC $\left(\mathrm{Kg} / \mathrm{m}^{2}\right)$ & $27,60 \pm 5,34$ & $27,74 \pm 4,70$ & 0,473 & $27,65 \pm 5,11$ \\
\hline PCB & $13,11 \pm 6,62$ & $17,29 \pm 7,30$ & $0,006^{\star \star}$ & $14,52 \pm 7,10$ \\
\hline CB & $29,66 \pm 4,58$ & $28,76 \pm 4,53$ & 0,524 & $29,35 \pm 4,56$ \\
\hline PCT & $16,84 \pm 7,34$ & $22,14 \pm 7,60$ & $0,002^{* *}$ & $18,63 \pm 7,80$ \\
\hline CMB & $24,89 \pm 3,89$ & $23,10 \pm 3,70$ & $0,041^{*}$ & $24,28 \pm 3,90$ \\
\hline
\end{tabular}

* Representa significância estatística no nível $p<0,05 .{ }^{*}$ Representa significância estatística no nível $p<0,01 .{ }^{* * *}$ Representa significância estatística no nível $p<0,001$. IMC: Índice de massa corporal; PCB: Prega cutânea biciptal; PCT: Prega cutânea tricipital; CMB: Circunferência muscular do braço; CB: Circunferência do braço.

Verificou-se, para as variáveis peso e estatura, maiores médias para o sexo masculino. Para a variável idade, observou-se diferença estatística com maiores médias expressadas para o sexo feminino. As variáveis PCB e PCT também apresentaram médias elevadas para o sexo feminino. Já para a variável $\mathrm{CMB}$, o sexo masculino apresentou maiores valores.

Soar (2015) avaliou 955 idosos frequentadores do Instituto Paulista de Geriatria e Gerontologia em São Paulo, mostrando a prevalência de fatores de risco cardiovasculares de acordo com o sexo e grupo etário, avaliando dentre os fatores de risco o excesso de peso e a adiposidade abdominal. As prevalências de excesso de peso foram de $47,40 \%$ para idosas e $36,12 \%$ para idosos, já para adiposidade abdominal foram, respectivamente de $76,16 \%$ para idosas e $41,06 \%$ para idosos.

Lavie et al. (2013) demonstraram em uma revisão sistemática, que estudos relatam um paradoxo a respeito da obesidade, seja ela medida por IMC, por percentual de gordura ou por obesidade central (circunferência de cintura), onde pessoas com sobrepeso e obesos apresentam um melhor prognóstico se comparados com pacientes com IC eutróficos ou com baixo peso. Porém, mesmo assim, esta revisão relatou que a hipertensão arterial sistêmica (HAS) e a doença arterial coronariana (DAC) são causas frequentes da insuficiência cardíaca (IC), tendo a 
obesidade como importante fator de risco, gerando numerosos efeitos adversos à saúde em geral e, particularmente, à saúde cardiovascular.

Bae et al. (2013) ressaltam que a composição corporal é constituída de fatores como massa muscular e massa gorda, sendo que estes marcadores mudam continuamente e estão diretamente relacionados ao processo de envelhecimento quando comparados a outros fatores. Mudanças na composição corporal das pessoas são marcadores de risco importantes ao desenvolvimento de doenças crônicas, principalmente com o avançar da idade. Monteiro et al. (2014) nos trazem também que essas mudanças na composição corporal, correlacionadas com a incidência de doenças crônicas, devem ter uma atenção primária no cuidado com a saúde.

Os pacientes da atual pesquisa não obtiveram diferenças estatísticas para a variável IMC em relação ao sexo, todavia, observou-se que os valores de média eram de $27,60 \mathrm{mg} / \mathrm{m}^{2}$ para homens e $27,74 \mathrm{~kg} / \mathrm{m}^{2}$ para mulheres, em $47,3 \%$ dos casos, 0 que se considera um nível de sobrepeso pela classificação de composição corporal segundo a Organização Mundial da Saúde (1995).

Estudos epidemiológicos já demonstraram uma relação entre IMC elevado e risco de mortalidade cardiovascular. Ainda, as doenças cardiovasculares foram responsáveis por $29,4 \%$ de todas as mortes registradas no país em um ano, isso significa que mais de 308 mil pessoas faleceram por esse tipo de patologia. A frequente incidência desse problema coloca o Brasil em um lugar de destaque entre os 10 países com maior índice de mortes por doenças cardiovasculares no mundo. (BRASIL, 2011; RUIZ-MONTERO et al., 2014).

Zomer et al. (2017) acompanharam anualmente, por 10 anos consecutivos, pacientes entre 30 e 74 anos, livres de doenças cardiovasculares pré-existentes e também acompanharam os fatores de risco destas patologias, a fim de calcular o risco de DCV. Os autores demonstraram que a perda de peso em pacientes ao longo dos anos associou-se à uma diminuição em termos de complicações cardiovasculares e pode proporcionar o ganho de anos de vida nestas pessoas.

O teste de associação Spearman foi realizado entre as variáveis quantitativas Idade, Peso, Estatura, IMC, PCB, CB, PCT e CMB. Os dados obtidos no teste estão expressos abaixo, na tabela 4 . Os dados foram analisados, avaliados pelos valores de "r", com grau de significância $p<0,05$ e $p<0,01$.

Tabela 4. Valores de "r" resultantes através do teste de associação de Spearman entre as variáveis quantitativas da amostra.

\begin{tabular}{cccccccc}
\hline Variáveis & Peso & Estatura & IMC & PCB & CB & PCT & CMB \\
\hline Idade & $-0,271^{*}$ & $-0,270^{*}$ & $-0,106$ & $-0,021$ & $-0,354^{* *}$ & $-0,015$ & $-0,344^{* *}$ \\
Peso & & $0,468^{* *}$ & $0,790^{* *}$ & $0,352^{* *}$ & $0,579^{* *}$ & $0,217^{*}$ & $0,544^{* *}$ \\
Estatura & & & $-0,088$ & $-0,162$ & $0,308^{* *}$ & $-0,259^{*}$ & $0,381^{* *}$ \\
IMC & & & & $0,534^{* *}$ & $0,520^{* *}$ & $0,408^{* *}$ & $0,406^{* *}$ \\
PCB & & & & & $0,471^{* *}$ & $0,789^{* *}$ & 0,058 \\
CB & & & & & & $0,376^{* *}$ & $0,683^{* *}$ \\
PCT & & & & & & $-0,139$ \\
\hline
\end{tabular}

* Representa significância estatística no nível $p<0,05$. ${ }^{* *}$ Representa significância estatística no nível $\mathrm{p}<0,01$. Valores de $\mathrm{r}: 0<\mathrm{r}<0,240=$ correlação nula; $0,250<r<0,490=$ correlação fraca; $0,500<r<$ $0,740=$ correlação moderada; $0,750<r<1=$ correlação forte; $0>r>-0,240=$ correlação nula inversamente proporcional; $-0,250>r>-0,490$ = correlação fraca inversamente proporcional; -0,500 > $r>-0,740=$ correlação moderada inversamente proporcional; $-0,750>r>-1=$ correlação forte inversamente proporcional. IMC: Índice de massa corporal; PCB: Prega cutânea biciptal; PCT: Prega cutânea tricipital; CMB: Circunferência muscular do braço; CB: Circunferência do braço. 
Os resultados do teste mostraram uma associação $(p<0,05)$ inversamente proporcional entre as variáveis Peso e Idade, onde quanto menor a idade, maior se mostrou o peso dos indivíduos. O mesmo se deu para a variável estatura em relação à idade, onde quanto menor a idade, maior se mostrou a estatura dos indivíduos analisados. Uma associação inversamente proporcional também foi observada entre as variáveis $\mathrm{CB}$ e idade, onde quanto menor a idade, maior foi observada a variável $\mathrm{CB}$, ou seja, ao passar da idade, a circunferência do braço diminuiria nestes pacientes. Ainda, quanto menor fora a estatura dos indivíduos, maiores foram os valores de PCT.

Pierine et al. (2009) demonstraram que o desuso da musculatura e o sedentarismo, cuja prevalência é elevada em idosos, também levam à redução da massa muscular (REIS J et al., 2014; ALMEIDA S et al., 2013). Sampaio et al. (2017) relataram que em relação à $\mathrm{CMB}$, a $\mathrm{PCT}$ reflete a gordura subcutânea, enquanto 0 perímetro do braço (PB) leva em conta o diâmetro do úmero, dos músculos esqueléticos e da gordura que cobrem o membro, demonstrando, assim, alterações na massa magra e gordura. É importante ressaltar que há uma diminuição mais acentuada da massa muscular, em membros, nos idosos.

A variável Peso obteve associação $(p<0,01)$ com as variáveis Estatura, IMC, PCB, CB, e CMB e também com a variável PCT $(p<0,05)$. Os dados mostram que quanto maior o peso, maiores os valores expressados nas demais variáveis citadas. Isso mostra que nestes pacientes, portadores de doenças cardiovasculares, os marcadores antropométricos relacionados principalmente ao peso, CB, PCB, e CMB, se mostraram maiores. Estes marcadores estão relacionados com a composição corporal, mostrando que nestes pacientes o acúmulo de gordura corporal possivelmente pode ser maior. Observa-se também, para $C B$, além da associação com a variável peso, uma associação $(p<0,01)$ com as variáveis estatura, IMC, e PCB. Nestes pacientes, quanto maior era a circunferência do braço, maior também era a prega cutânea bicipital (PCB).

Para a variável PCT, além da associação com a variável peso $(p<0,05)$, foi observada uma associação $(p<0,01)$ com as variáveis IMC, PCB e CB. Assim como a variável $\mathrm{PCB}$, a variável $\mathrm{PCT}$ também se mostrou correlacionada à CB. Além disso, CMB também mostrou associação $(p<0,01)$ com as variáveis IMC e CB.

$\mathrm{Na}$ tabela 5, estão descritos os valores pela análise de variância (ANOVA-one way) entre a incidência do número de patologias e/ou intervenções, associadas com a média e desvio padrão das variáveis antropométricas dos indivíduos.

Tabela 5. Valores expressos em média e desvio padrão para as variáveis quantitativas Idade, Peso, Estatura, IMC, PCB, CB, PCT e CMB, em relação ao número de patologias observados na amostra.

\begin{tabular}{|c|c|c|c|c|}
\hline \multirow[b]{2}{*}{ Variáveis } & \multicolumn{4}{|c|}{ Número de casos patológicos/intervenções } \\
\hline & Uma & Duas & Três & p-valor \\
\hline Idade & $72,06 \pm 11,9$ & $67,50 \pm 11,9$ & $69,68 \pm 10,3$ & 0,292 \\
\hline Peso & $74,66 \pm 11,8$ & $83,55 \pm 17,6$ & $74,45 \pm 16,3$ & 0,098 \\
\hline Estatura & $170,27 \pm 9,5^{\mathrm{a}}$ & $169,00 \pm 9,2^{a}$ & $162,85 \pm 6,8^{b}$ & $0,009^{\star *}$ \\
\hline IMC & $25,73 \pm 3,6^{a}$ & $29,31 \pm 5,1^{b}$ & $27,68 \pm 5,9^{a b}$ & $0,018^{*}$ \\
\hline PCB & $12,36 \pm 7,3$ & $16,19 \pm 7,9$ & $14,86 \pm 4,4$ & 0,094 \\
\hline CB & $28,20 \pm 3,9$ & $30,73 \pm 4,2$ & $28,79 \pm 5,3$ & 0,066 \\
\hline PCT & $15,60 \pm 7,4^{a}$ & $19,38 \pm 8,2^{\mathrm{ab}}$ & $21,59 \pm 6,2^{b}$ & $0,016^{*}$ \\
\hline $\mathrm{CMB}$ & $23,62 \pm 3,4$ & $25,13 \pm 4,3$ & $23,87 \pm 3,6$ & 0,262 \\
\hline
\end{tabular}

${ }^{*}$ Representa significância estatística no nível $p<0,05 .{ }^{* *}$ Representa significância estatística no nível $p<0,01$. Letras iguais representam médias iguais. Letras diferentes representam médias diferentes. IMC: Índice de massa corporal; PCB: Prega cutânea biciptal; PCT: Prega cutânea tricipital; CMB: Circunferência muscular do braço; CB: Circunferência do braço. 
De acordo com a tabela 5, a variável IMC mostrou-se com maior média para indivíduos com até duas patologias e/ou intervenções. No entanto, observa-se também que os pacientes que apresentaram até 3 patologias e/ou intervenções, apresentavam valores de média iguais tanto para duas quanto para uma patologia e/ou intervenções, portanto, possuir uma ou mais patologias e/ou intervenções pode apresentar igualdade no IMC. O peso dos pacientes que apresentavam duas patologias associadas mostrou-se com maior média, todavia, esta variável não diferiu entre os pacientes que possuíam uma ou três patologias. Ainda, foi verificado que os pacientes que apresentaram três tipos de patologias e/ou intervenções associadas, apresentavam maiores valores de PCT em relação aos pacientes com duas ou apenas uma patologia e/ou intervenção. Sendo assim, pacientes com três patologias associadas possuíam maior acúmulo de gordura corporal na prega cutânea tricipital.

Ainda, na tabela 5, verificou-se para a variável estatura que os pacientes que apresentam três patologias e/ou intervenções possuíam menor estatura em relação aos que apresentaram até uma ou duas patologias e/ou intervenções.

\section{CONSIDERAÇÕES FINAIS}

Dentre os pacientes da amostra, a maior incidência nos indivíduos era a de hipertensão Arterial, seguida respectivamente de dislipidemias, angioplastia, insuficiência cardíaca, infarto agudo do miocárdio e doença arterial coronariana. Ainda, os resultados mostraram que o número de patologias entre os sexos se apresentaram igualmente quando se trata da presença de uma, duas ou três patologias associadas, mesmo a amostra sendo composta em sua maioria por homens. Quanto mais a idade dos indivíduos avança, menor é o peso dos mesmos, sendo também menores os valores para CB. Ainda, indivíduos com menor estatura demonstraram possuir maiores números para PCT.

Para as variáveis índice de massa corporal e peso foi verificado maiores médias para quem possuía até duas patologias e/ou intervenções associadas. No entanto, possuir uma ou mais patologias e/ou intervenções pode apresentar igualdade no IMC e no peso dos indivíduos avaliados. As doenças cardiovasculares fazem parte das principais causas de morbidade e mortalidade no mundo todo, e sabe-se que mudanças de peso e maior acúmulo de gordura corporal aumentam o risco para o desenvolvimento desse tipo de patologia. Sendo assim, o conhecimento, para entender melhor como se mostram os indivíduos portadores deste tipo de doença, no quesito composição corporal, é de suma importância para que se possa proporcionar a melhora do estado nutricional e também para traçar estratégias de prevenção e/ou tratamento dessas doenças, nestes indivíduos.

Sugere-se que novas pesquisas sejam realizadas para mostrar como os marcadores de composição corporal se mostram em diferentes patologias cardiovasculares, a fim de traçar um melhor perfil epidemiológico desses pacientes. É importante que essas novas pesquisas sejam realizadas em diferentes regiões, para que se possa compreender melhor como se mostram os pacientes portadores de doenças cardiovasculares em diferentes regiões demográficas. Por fim, a utilização de outras ferramentas de análise de composição corporal pode proporcionar uma análise mais completa dos dados coletados. Ressalta-se ainda que, é de suma importância ao nutricionista o conhecimento destas informações a respeito dos pacientes e sua epidemiologia, afim de proporcionar ações efetivas no tratamento preventivo e na intervenção em busca da melhora do estado de saúde dos indivíduos. 


\section{REFERÊNCIAS}

ALMEIDA, S.N.; MENEZES, T.N.; MELO, R.L.P.; PEDRAZA, D.F.; Força de preensão manual e flexibilidade e suas relações com variáveis antropométricas em idosos. Revista da Associação Médica Brasileira. 2013; 59(2):128-135.

ARAÚJO, SP, et al. Mulheres na atenção primária à saúde: exercício físico, estilo de vida e fatores de risco cardiovascular. Revista Eletrônica de Comunicação, Informação \& Inovação em Saúde, Rio de Janeiro, v. 11, n. 3, sep. 2017.

BAE, C.Y.; KANG, Y.G.; SUH, Y.S.; HAN, J.H.; KIM, S.S.; SHIM, K.W.; A model for estimating body shape biological age based on clinical parameters associated with body composition. Clin Interv Aging. 2013;8:11-18.

BRASIL. MINISTÉRIO DA SAÚDE. Doenças cardiovasculares causam $30 \%$ das mortes no país. São Paulo, 2011. Disponível em: < http://www.brasil.gov.br/saude/2011/09/doencas-cardiovasculares-causam-quase30-das-mortes-no-pais>. Acesso em:05/11/2019.

CESARINO, C..B.; CIPULLO, J.P.; MARTIN, J.F.V.; CIORLIA, L.A.; GODOY, M.R.P.; CORDEIRO, J.A.; et al. Prevalence and Sociodemographic Factors in a Hypertensive Population in São José do Rio Preto, São Paulo, Brazil. Arq Bras Cardiol [Internet] 2008 [cited 2018 Jan 08]; 91(1):31-35. Available from: http://www.scielo.br/pdf/abc/v91n1/en a05v91n1.pdf [ Links ]

COELHO, J.C. et al. A hipertensão arterial é causa subjacente de morte avaliada na autópsia de indivíduos. Revista da Escola de Enfermagem da USP, v. 53, 2019.

GEER, E.B.; SHEN, W.: Diferenças de gênero na resistência à insulina, composição corporal e balanço energético. [Revisão] [156 refs]. Medicina de gênero. 2009, 6 (Suppl): 75.

INSTITUTO BRASILEIRO DE GEOGRAFIA E ESTATÍSTICA (IBGE). Pesquisa de Orçamentos Familiares 2008-2009. Rio de Janeiro: Ministério do Planejamento, Orçamento e Gestão; 2010.

LARSSON, B.; SVARTSUDD, K. L.; WELIN, W.L.; BJORNTORP, P.; TIBBLIN, G.: Distribuição do tecido adiposo abdominal, obesidade e risco de doença cardiovascular e morte: 13 anos de acompanhamento dos participantes no estudo de homens nascidos em 1913. British Medical Journal Clinical Research Ed. 1984, 288: 14011404. 10.1136 / bmj.288.6428.1401.

LAVIE, C.J.; ALPERT, M.A.; ARENA, R.; MEHRA, M.R.; MILANI, R.V.; VENTURA, H.O.: Impact of obesity and the obesity paradox on prevalence and prognosis in heart failure. JACC Heart Fail. 2013;1(2):93-102. doi: 10.1016/j.jchf.2013.01.006.

LEE, C.M.; HUXLEY, R.R.; WILDMAN, R. P.; WOODWARD, M.: Índices de obesidade abdominal são melhores discriminadores de fatores de risco cardiovascular do que o 
IMC: uma meta-análise. J Clin Epidemiol. 2008, 61: 646-653. 10.1016 / j.jclinepi.2007.08.012.

MARGARET, A.; SHIUN, D.H.: Seis razões pelas quais a relação cintura-estatura é um indicador global rápido e eficaz para os riscos à saúde da obesidade e como seu uso poderia simplificar a mensagem internacional de saúde pública sobre a obesidade. Int J Food Sci Nutr. 2005, 56 (5): 303-307. 10.1080 / 09637480500195066.

MAURIĖGE, P.; IMBEAULT, P.; PRUD‘HOMME, D.; TREMBLAY, A.; NADEAU, A. \& DESPRÉS, J.P.; Subcutaneous adipose tissue metabolism at menopause: importance of body fatness and regional fat distribution. $J$ Clin Endocrinol Metab 2000;85(7):2446-2454. 14.

MELANSON, K.J.; MCINNIS, K.J.; RIPPE, J.M.; BLACKBURN, G.; WILSON, P.F.: Obesidade e risco de doença cardiovascular: atualização de pesquisa. [Revisão] [50 refs]. Cardiologia em revisão. 2001, 9: 202-207. 10.1097 / 00045415-20010700000005.

MENDIS, S.; PUSKA, P.; NORRVING, B.: Global atlas on cardiovascular disease prevention and control: policies, strategies and interventions. Geneva: World Health Organization; World Heart Federation; World Stroke Organization; 2011.

MOZAFFARIAN, D.; BENJAMIN, E.J.; GO A.S.; ARNETT, D.K.; BLAHA, M.J.; CUSHMAN, M. et al. Heart disease and stroke statistics-2015: update a report from the American Heart Association. Circulation. 2015;131(4):e29-322. DOI: 10.1161/CIR.0000000000000152.

NORDSTRAND, N.; GJEVESTAD, E.; DINH1, K.N.; HOFSO, D.; ROISLIEN, J.; SALTVEDT1, E.; HJELMESAETH, I. O. S.J.; A relação entre várias medidas de obesidade e rigidez arterial em pacientes com obesidade mórbida. BMC Cardiovascular Disorders 2011, 11:7 http://www.biomedcentral.com/1471$\underline{2261 / 11 / 7}$.

OGDEN, C.L., et al. Prevalence of obesity and trends in Body Mass Index among US children and adolescents, 1999-2010. JAMA. Vol. 307. Num. 5. p. 483-490. 2012.

PIERINE. D.T.; NICOLA, M.; OLIVEIRA, E.P.: Sarcopenia: alterações metabólicas e consequências no envelhecimento. Rev Bras Ciênc Mov 2009; 17(3):96-103.

REIS, J.W.M.; CARNEIRO, J.A.O.; COQUEIRO, R.S.; SANTOS, K.T.; FERNANDES, M.H.; Pré-fragilidade e fragilidade de idosos residentes em município com baixo Índice de Desenvolvimento Humano. Rev. Latino-Am. Enfermagem 2014; 22(4):654-6152.

RUIZ-MONTERO, P. J.; CASTILLO-RODRIGUEZ, A.; MIKALAČKI, M.; NEBOJSA, Č.; KOROVLJEV, D. 24-weeks Pilates-aerobic and educative training to improve body fat mass in elderly Serbian women. Clinical interventions in aging. Vol. 9. p. 243. 2014.

SAMPAIO, L.S., et al. Indicadores antropométricos como preditores na determinação da fragilidade em idosos. Ciência \& Saúde Coletiva, v. 22, p. 4115-4124, 2017. 
SANCHES, F. L., \& BACCHI, M. K.; (2017). Resumo: Avaliação antropométrica de pacientes adultos e idosos cardiopatas hospitalizados. PECIBES, supl. 2, 24, 2016. Perspectivas Experimentais e Clínicas, Inovações Biomédicas e Educação em Saúde (PECIBES), 2(2 Supl. 2).

SANTANA, G. D. J.; VILA-NOVA, T. M. S.; COSTA, J. O.; VÁZQUEZ, C. M. P., \& BARBOSA, K. B. F.: (2016, November). Risco cardiometabólico em indivíduos jovens e clinicamente saudáveis: associação com o estado nutricional. Em Congresso Internacional de Atividade Física, Nutrição e Saúde (No. 1).

SCHNEIDER, H.J.; FRIEDRICH, N.; CLOTSCHE, J.; PIEPER, L.; NAUCK, M.; JOHN, U., et al: $O$ valor preditivo de diferentes medidas de obesidade para eventos cardiovasculares incidentes e mortalidade. Jornal de Endocrinologia Clínica e Metabolismo. 2010, 95: 1777-1785. 10.1210 / jc.2009-1584.

SOAR, C. Prevalência de fatores de risco cardiovascular em idosos não institucionalizados. Revista Brasileira de Geriatria e Gerontologia, Rio de Janeiro, 18(2): 385-395 2015.

TATSUKAWA, M.; KUROKAWA, M.; TAMARI, Y.; YOSHIMATSU, H. \& SAKATA T. Regional fat deposition in the legs is useful as a presumptive marker of antiatherogenesity in Japanese. PSEBM 2000;223:156-162.

The IDF consensus worldwide definition of metabolic syndrome. International Diabetes Federation, 2006 [Acesso em 2019 Novembro 8]. Disponível em: http://www.idf.org.

VAN, D.; KROMHOUT, D.; GELEIJNSE, J.M.; BOER, J. M., VERSCHUREN, W.M.: Índice de massa corporal e circunferência da cintura em ambos os sexos por 10 anos e o risco de doença cardiovascular fatal e não fatal: estudo realizado em 20.000 homens e mulheres holandeses com idades entre 20-65 anos. Revista Europeia de Prevenção e Reabilitação Cardiovascular. 2009, 16: 729-734. 10.1097 / HJR.0b013e328331dfc0.

WANG, W.; ZHAO, D.; JY, S.; LIU, J.; QIN, L. P., et al: Valor preditivo de medidas combinadas de índice de massa corporal e circunferência da cintura para o risco de doença cardiovascular. Revista Chinesa de Cardiologia. 2008, 36: 655-658.

WANG, Y.; MONTEIRO, C.A.; POPKIN, B.M.: Trends of obesity and underweight in older children and adolescents in the United States, Brazil, China and Russia. The American Journal of Clinical Nutrition. Vol. 75. Num. 6. p. 971-977. 2002.

WORLD HEALTH ORGANIZATION. A global brief on hypertension: silent killer, global public health crisis. World Health Day 2013. Geneva: World Health Organization; 2013.

WORLD HEALTH ORGANIZATION. Physical status: the use and interpretation of anthropometry. Geneva: World Health Organization; 1995. (Technical Report Series, 854). 
YUSUF, S.; HAWKEN, S.; OUNPUU, S.; BAUTISTA, L.; FRANZOSI, M.G.; COMMERFORD, P., et al: Obesidade e o risco de infarto miocárdico em 27.000 participantes de 52 países: um estudo do caso-controle. Lanceta. 2005, 366: 16401649. 10.1016 / S0140-6736 (05) 67663-5.

ZOMER, E.; LEACH, R.; APARADOR, C.; LOBSTEIN, T .; MORRIS, S.; JAMES, W.P.; FINER, E. N.: Eficácia e custo-efetividade de intervenções que causam perda de peso e reduzem o risco de doença cardiovascular, Diabetes Obes Metab , 2016 . doi: $10.1111 /$ dom. 12792 . 